\title{
Study on the Effect of Clinical Treatment of Vaginitis in Obstetrics and Gynecology
}

\author{
Hua Wei, Xiangyan Feng, Sale Zhang, Mingxian Guo * \\ Xi'an Peihua University, Xi'an City, Changan District China Road No. 1, 710125
}

Keywords: vaginitis; Obstetrics and Gynecology; combination of traditional Chinese and Western medicine

\begin{abstract}
To investigate the clinical effect of Obstetrics and Gynecology vaginitis. Methods: A total of 120 vaginitis patients in our hospital in June 2016 -2017 were involved in the study, randomly divided into observation group and control group, 60 cases in each group, the control group was treated with western medicine treatment, using oral medicine way, observation group were treated with traditional Chinese and Western medicine with treatment, the patients were treated with oral form of antibiotics, and the treatment of two groups of patients were analyzed. Results: the therapeutic effect of the patients in the observation group was better than that of the control group, and the difference was statistically significant $(\mathrm{P}<0.05)$. Conclusion: in the process of the treatment of vaginitis, the combination of traditional Chinese and Western medicine is effective and worthy of clinical application [1].
\end{abstract}

Vaginitis is a common clinical disease in obstetrics and gynecology department. In healthy condition, there will be adequate aerobic bacteria and anaerobic bacteria in the vagina, so as to ensure the balance of vaginal flora. If the balance of the bacteria in the vagina is broken, the pathogen is easily intruded into the vagina, causing inflammation. In clinic, common diseases include bacterial vaginitis, senile vaginitis and candidal vaginitis. Among them, the number of bacterial vaginitis and candidal vaginitis is the largest. Vaginitis patients with abnormal leucorrhea, the vagina will secrete a large number of foreign bodies, the vagina will appear pruritus and prickle sensation around the vagina. Although this kind of disease does not pose a threat to the life of the patient, it is easy to relapse after treatment and has a great impact on the life of the patient. This study analyzes the combination of traditional Chinese and Western medicine in the treatment of vaginitis, and the effect is prominent.

\section{Data and Methods}

This study analyzed 120 cases of vaginitis in our hospital. The patients were randomly divided into the observation group and the control group, with 60 cases in each group. Renal insufficiency can not be involved in this study, if the patient has systemic disease, and the western medicine nationals can not be involved in the study. The minimum age of the control group was 20 years, the maximum age of 60 years, the average age of 32 years. The minimum age of the observation group was 18 years old, the maximum age of 59 years, the average age of 32 years [2].

The patients in the control group were treated with conventional western medicine. Before treatment, the vaginal secretions of the patients were examined to determine the type of bacterial infection. If the patient has bacterial vaginitis, the patient should be treated with metronidazole and Tinidazole, such as anti anaerobes. If patients with candidal vaginitis, it should stop the use of all kinds of antibiotics and corticosteroid medications, with miconazole suppository and Clotrimazole Suppositories agent for local treatment, if the patient's condition or repeated episodes, then combined with drugs for the treatment of itraconazole and fluconazole etc. If the patient has trichomonad vaginitis, it should be treated with local treatment, while $0.5 \%$ acetic acid and $1 \%$ lactic acid should be used to clean the vagina.

The observation group was treated with traditional Chinese medicine on the basis of the treatment of Western medicine. With Longdanxiegan pills, leucorrhea pill and Xiaoyao Pill for 
treatment.

Two groups of patients were treated for three months.

\subsection{Evaluation of curative effect}

The patients in the observation group and the control group were treated for three months continuously, and the effect of the patients was analyzed.

The clinical symptoms of the patients were all disappeared, and there was no pathogenic bacteria after the etiological examination. After the treatment, the symptoms were not cured for one year [3].

The patients did not feel abnormal, and the clinical symptoms were improved to be effective.

The symptoms of the patients improved, but they felt abnormally better.

There was no improvement in the symptoms during the treatment of the patients, and even the aggravation of the disease was ineffective.

\subsection{Statistical method}

In this study, SPSS 20 was used to analyze the related data, the standard deviation was used to analyze the data, and the percentage was used to analyze the counting data.

\section{Results}

Table 1 Comparison of the treatment effect between the observation group and the control group

\begin{tabular}{ccccccc}
\hline Group & $\begin{array}{c}\text { The } \\
\text { number } \\
\text { of cases }\end{array}$ & Cure & Effective & Effective & Invalid & $\begin{array}{c}\text { Total effective } \\
\text { rate }\end{array}$ \\
\hline $\begin{array}{c}\text { Observation } \\
\text { group }\end{array}$ & 60 & 25 & 28 & 6 & 1 & $98.3 \%$ \\
Control group & 60 & 18 & 23 & 12 & 7 & $88.3 \%$ \\
\hline
\end{tabular}

\section{Discussion}

A healthy woman's vagina is characterized by an anatomical structure, a large number of pathogens invading, the vagina producing a barrier, and self defense. In healthy women, the anterior and posterior walls of the vagina are close to each other, and the vaginal orifice is in a closed state. The acidic and alkaline state of the vagina is in a state of equilibrium. Vaginal epithelial cells proliferate under the influence of estrogen, which can prevent the pathogen from propagating in the vagina. If the natural defense function of the vagina is greatly damaged, when the pathogen invades, it will lead to the imbalance of acid-base in the vagina, providing conditions for the growth and reproduction of bacteria, leading to vaginitis. Vaginitis is divided into many types. The main types are bacterial vaginitis, senile vaginitis and candidal vaginitis. Vaginitis is one of the diseases that cannot be cured at once. It is very stubborn and has great interference to the life of patients. Therefore, the clinical treatment of vaginitis has caused widespread concern among medical staff [4].

Vaginal microecology constitutes a variety of components, when any kind of component imbalance can lead to vaginal microecological imbalance. Vaginal microecological imbalance can lead to vaginal infections. In the microecological community, the conditional pathogenic bacteria can be produced, such as the pathogenic microbes of vaginal infection, such as Escherichia coli, Candida, trichomonas and so on. The pathogenic bacteria of bacterial vaginitis are mainly Gardiner, Streptococcus, Campylobacter and so on. Their concentration in vagina is relatively high, which leads to imbalance of vaginal microecology and abnormal increase of microorganism, leading to vaginitis. Vaginal microbiosis in patients with bacterial vaginitis is 100 times higher than that in healthy people. Moreover, with the decrease of Lactobacillus in vagina, the amount of hydrogen peroxide produced is insufficient. In patients undergoing bacterial vaginitis, it is found that the $\mathrm{pH}$ value of vaginal secretions is significantly improved, the concentration of various enzymes in the 
body is increasing, and the concentration of prostaglandins is also improving. When the enzyme substances are elevated, the defense mechanism of the body will be greatly destroyed, causing the microbes in the vagina to enter the genital tract and produce infection. But there are also reports that inflammatory factors in patients with bacterial vaginitis will change, which will directly lead to the microbial community imbalance, which will lead to the increase of the number of pathogenic bacteria and the infection of Trichomonas, mycoplasma and chlamydia. If the other pathogenic microbes are infected, it can cause inflammation. If other pathogens are infected with microbes, they will also cause bacterial vaginitis, which will cause various complications, such as endometritis and pelvic inflammatory disease, which will cause serious consequences for mother and infant. After the patient has bacterial vaginitis, the incidence of infertility in the fallopian tube is significantly higher than that in the healthy population, and it will also lead to the occurrence of early abortion.

Mycotic vaginitis, also known as Candida vaginitis, is a common vaginitis. In the taste microecological environment, Candida is the main pathogen. The related literature points out that $30 \%$ of women have Candida in the vagina. In the normal microecological environment, the content of Candida is not much, and it will not cause the infection. If the immunity of the patient drops, the Candida albicans will reproduce a lot, which will cause the infection. The blood glucose of patients will rise sharply, leading to a great change in the glucose metabolism of leukocytes, leading to the change of neutrophils, the lack of phagocytosis of the central granulocytes, the decrease of immunoglobulin and the lack of transformation rate of lymph. In the case of hyperglycemia, the synthesis of glycogen in the epithelial cells of the vagina can be promoted, resulting in a decrease in the $\mathrm{pH}$ value of the vagina and the growth of a large number of yeast.

There are a large number of microorganisms in the vagina of women's health, but all kinds of microorganisms and restrict each other, in a harmonious environment, vaginal micro ecological environment in a state of balance, lactobacillus can maintain vaginal $\mathrm{pH}$ value, so as to prevent the propagation of all kinds of bacteria, the vaginal cleaning effect. The vulva of the bacterial vaginitis will appear pruritus, the secretion has the odor, the number of lactobacilli in the vagina is reduced. Fungal vaginitis can cause serious vulva pruritus and abnormal leucorrhea, after the treatment of recurrent, should use a combination of oral and external, combining traditional Chinese and Western medicine for the treatment of the way [5].

Normal vagina in hydrogen peroxide containing large amounts of lactic acid bacteria, when the patients had bacterial vaginitis, the number of patients with vaginal Lactobacillus will be a sharp decline, the number of anaerobic bacteria will increase dramatically, then the body will appear in patients with mixed infection of endogenous, patients often appear fishy secretions increase, the vulva will have the feeling of itching, vaginal mucosa will produce congestion. The type of Candida vaginitis is common, then the patient's vaginal acidity is very strong, Candida albicans is easy to grow and reproduce in the vagina, if patients suffering from diabetes, or in patients with pregnancy, in the use of a large number of spectrum antibiotics and various immune drugs, patients can secrete tofu like secretions the patient will appear, the vulva pruritus, dysuria and frequency etc. When patients with Trichomonas vaginitis, then a large number of Trichomonas vaginalis will multiply in the patient's vagina, this kind of bacteria growing in the neutral environment, and at the same time also love moist, seizures and other types of vaginitis patients, leucorrhea yellow green, and there will be pus and irritation from the patient's discharge from the vagina, vulva pruritus patients, serious patients will lead to urinary tract infection and infertility. If ovarian function in the elderly will lead to recession, senile vaginitis, the female hormone level will be relatively poor, then the patients with vaginal alkaline, and vaginal structure will change, the body's resistance will decline, a large number of anaerobic bacteria breeding in patients with vagina. The cause of female vaginitis is the vulva dysplasia of the patient. There are a lot of foreign bodies in the vagina. The level of estrogen secretion is reduced, leading to vaginal infection. The main bacteria are Streptococcus and Staphylococcus.

Vaginitis is the general name of various kinds of inflammation of the vaginal mucosa, and it is a common disease of reproductive system infection. Oxidative stress causes the production of 
vaginitis. With the development of medical technology, free radicals have been studied in all kinds of diseases. By studying the oxidative stress, we can well analyze the pathogenesis of various diseases. In the study of the pathogenesis of vaginitis, oxidative stress will also be analyzed. In the patients with bacterial vaginitis, the lactobacillus and vaginal $\mathrm{pH}$ values and the contents of various enzymes in the patients were analyzed. It was found that the content of Lactobacillus and PH in all vaginitis patients showed a decreasing trend, and the activity of CAT and SOD decreased. Therefore, in the case of oxidative stress, vaginitis often occurs, and the vaginitis and oxidant factors are out of balance. When the free radicals in the body increase, the cells will produce severe oxidative stress, resulting in mitochondrial disorder, resulting in a large number of cell apoptosis, resulting in cell damage. When the virulence of the strain in the vagina of the patient is enhanced, the expression of aspartic protease will also change. In the study of gene sequencing, the sequencing of various pathogenic microbes has been gradually completed. In the study of virulence factors, it is found that Candida albicans secretes acid protease, which is very toxic and can hydrolyze extracellular proteins. Aspartic protease can degrade various host proteins in the vagina. After the protein is degraded, the environment in the vagina is very suitable for the growth of Candida, resulting in inflammation of vaginal epithelial cells.

In the process of vaginitis treatment, antibiotics are often used. Local or systemic medication is used to effectively inhibit the reproduction of pathogens and clean the vulva everyday. Patients often are infected by more than two pathogens. If they are treated with western medicine alone, the effect is not very obvious, and patients often relapse after treatment. The use of a large number of antibiotics will result in dependence on the patient, and the adverse reactions will increase, which is not conducive to later treatment. Therefore, the combination of traditional Chinese and Western medicine in the way of citing treatment has achieved good results.

With the gradual improvement of people's living standards, people's awareness of health is also improving. In the clinical treatment of vaginitis, traditional Chinese medicine is used. In the treatment of vaginitis with oral medication, the general way is to take oral Chinese medicine, aiming at the dialectical way of traditional Chinese medicine, aiming at different types of vaginitis. The external treatment is mainly in the vagina or around the vagina. It can be treated by traditional Chinese medicine. In the treatment of vaginitis, it can be treated with Matrine vaginal effervescent Tablets, the effect is obvious. In the compound radix sophorae Sophora flavescens lotion, the main ingredients are Sophora flavescens, honeysuckle, white skin, and so on, can relieve the patient's inflammation. Combined use of internal and external treatment, the effective rate of treatment is also very high [6].

In the field of traditional Chinese medicine, the cause of vaginitis lies in the loss of the liver and kidney of the patient and kidney deficiency. In the treatment, it is used in the way of the remedy. Combined with internal and external treatment. If the patient is the type of spleen deficiency, it should be treated by the way of sterilizing and stopping itching, and using the principle of strengthening the spleen. Damp type if the patient is a general treatment method is used and the qingrelishi shajunzhiyang. If the patient is liver and kidney deficiency type, the method should be used to nourish liver and kidney. If the patient is kidney deficiency and dampness type, the treatment with sterilization antipruritic and warming kidney dampness.

In the present study, the patients in the observation group on the basis of Western medicine, also used the pill, Xiaoyao Pill and leucorrhea of traditional Chinese medicine treatment, contains a lot of gentian and gardenia and other drugs in Longdanxiegan pills, this kind of medicine has the effect of clearing liver and gallbladder. Leucorrhea pills contain angelica, Paeonia lactiflora, and other drugs, play the effect of clearing heat and dehumidification. Jiawei Xiaoyao Pill contains Radix Angelicae and Radix Bupleuri and other drugs, which can improve the effect of strengthening the spleen and nourishing blood.

In this study, the therapeutic effect of the observation group is better than that of the control group, which shows that the effect of traditional Chinese medicine is more valuable on the basis of the treatment of Western medicine. 


\section{References}

[1] Dong J. Study on clinical treatment of refractory obstetrics and gynecology hemorrhage[J]. China Modern Doctor, 2017.

[2] Cui G R. Clinical study on the Treatment of Chronic Pelvic Inflammatory Disease in Gynecology and Obstetrics[J]. World Latest Medicine Information, 2017.

[3] Tian A. Effect Analysis of Seamless Nursing Management in Nosocomial Infection in Obstetrics and Gynecology Department[J]. China Continuing Medical Education, 2017.

[4] Gaydos C A, Beqaj S, Schwebke J R, et al. Clinical Validation of a Test for the Diagnosis of Vaginitis. [J]. Obstetrics \& Gynecology, 2017, 130(1):181.

[5] Shi Y P. Analysis of the Effect of Metronidazole and Clindamycin in Patients with Bacterial Vaginosis[J]. World Latest Medicine Information, 2017.

[6] He H. Clinical treatment of vaginitis in gynecology clinic and the influencing factors of vaginitis[J]. Chinese Community Doctors, 2017. 